\title{
The prognostic value of right ventricular deformation imaging in early arrhythmogenic right ventricular cardiomyopathy
}

Citation for published version (APA):

Mast, T. P., Taha, K., Cramer, M. J., Lumens, J., van der Heijden, J. F., Bouma, B. J., van den Berg, M. P., Asselbergs, F. W., Doevendans, P. A., \& Teske, A. J. (2019). The prognostic value of right ventricular deformation imaging in early arrhythmogenic right ventricular cardiomyopathy. JACC: Cardiovascular Imaging, 12(3), 446-455. https://doi.org/10.1016/j.jcmg.2018.01.012

DOI:

10.1016/j.jcmg.2018.01.012

Document status and date:

Published: 01/03/2019

Document Version:

Publisher's PDF, also known as Version of Record (includes final page, issue and volume numbers)

Please check the document version of this publication:

- A submitted manuscript is the version of the article upon submission and before peer-review. There can be important differences between the submitted version and the official published version of record. People interested in the research are advised to contact the author for the final version of the publication, or visit the $\mathrm{DOI}$ to the publisher's website.

- The final author version and the galley proof are versions of the publication after peer review.

- The final published version features the final layout of the paper including the volume, issue and page numbers.

Link to publication

\section{General rights}

Copyright and moral rights for the publications made accessible in the public portal are retained by the authors and/or other copyright owners and it is a condition of accessing publications that users recognise and abide by the legal requirements associated with these rights.

- Users may download and print one copy of any publication from the public portal for the purpose of private study or research.

- You may not further distribute the material or use it for any profit-making activity or commercial gain

- You may freely distribute the URL identifying the publication in the public portal.

If the publication is distributed under the terms of Article $25 \mathrm{fa}$ of the Dutch Copyright Act, indicated by the "Taverne" license above, please follow below link for the End User Agreement:

www.tue.nl/taverne

Take down policy

If you believe that this document breaches copyright please contact us at:

openaccess@tue.nl

providing details and we will investigate your claim. 


\section{The Prognostic Value of Right Ventricular Deformation Imaging in Early Arrhythmogenic Right Ventricular Cardiomyopathy}

Thomas P. Mast, MD, PHD, ${ }^{\mathrm{a}, \mathrm{b}}, *$ Karim Taha, BSc, ${ }^{\mathrm{c} *}$ Maarten J. Cramer, MD, PHD, ${ }^{\mathrm{a}}$ Joost Lumens, $\mathrm{PHD}^{\mathrm{d}}{ }^{\mathrm{d}}$ Jeroen F. van der Heijden, MD, PHD, ${ }^{a}$ Berto J. Bouma, MD, PHD, ${ }^{e}$ Maarten P. van den Berg, MD, PHD, ${ }^{f}$ Folkert W. Asselbergs, MD, PHD, ${ }^{\mathrm{a}, \mathrm{g}, \mathrm{h}}$ Pieter A. Doevendans, MD, $\mathrm{PHD},{ }^{\mathrm{a}}$ Arco J. Teske, MD, $\mathrm{PHD}^{\mathrm{a}}$

\section{ABSTRACT}

OBJECTIVES The aim of this study was to investigate the prognostic value of echocardiographic deformation imaging in arrhythmogenic right ventricular cardiomyopathy (ARVC) to optimize family screening protocols.

BACKGROUND ARVC is characterized by variable disease expressivity among family members, which complicates family screening protocols. Previous reports have shown that echocardiographic deformation imaging detects abnormal right ventricular (RV) deformation in the absence of established disease expression in ARVC.

METHODS First-degree relatives of patients with ARVC were evaluated according to 2010 task force criteria, including RV deformation imaging $(n=128)$. Relatives fulfilling structural task force criteria were excluded for further analysis. At baseline, deformation patterns of the subtricuspid region were scored as type I (normal deformation), type II (delayed onset, decreased systolic peak, and post-systolic shortening), or type III (systolic stretching and large post-systolic shortening). The final study population comprised relatives who underwent a second evaluation during follow-up. Disease progression was defined as the development of a new 2010 task force criterion during follow-up that was absent at baseline.

RESULTS Sixty-five relatives underwent a second evaluation after a mean follow-up period of $3.7 \pm 2.1$ years. At baseline, 28 relatives (43\%) had normal deformation (type I), and 37 relatives (57\%) had abnormal deformation (type II or III) in the subtricuspid region. Disease progression occurred in $4 \%$ of the relatives with normal deformation at baseline and in $43 \%$ of the relatives with abnormal deformation at baseline $(p<0.001)$. Positive and negative predictive values of abnormal deformation were, respectively, $43 \%$ (95\% confidence interval: $27 \%$ to $61 \%$ ) and $96 \%$ (95\% confidence interval: $82 \%$ to $100 \%)$.

CONCLUSIONS Normal RV deformation in the subtricuspid region is associated with absence of disease progression during nearly 4-year follow-up in relatives of patients with ARVC. Abnormal RV deformation seems to precede the established signs of ARVC. RV deformation imaging may potentially play an important role in ARVC family screening protocols. (J Am Coll Cardiol Img 2019;12:446-55) @ 2019 by the American College of Cardiology Foundation.

\footnotetext{
From the a Department of Cardiology, Division Heart and Lungs, University Medical Center Utrecht, Utrecht, the Netherlands; bepartment of Cardiology, Catharina Hospital Eindhoven, Eindhoven, the Netherlands; 'University of Amsterdam, Amsterdam, the Netherlands; ${ }^{\mathrm{d} D e p a r t m e n t}$ of Biomedical Engineering, Cardiovascular Research Institute Maastricht, Maastricht University, Maastricht, the Netherlands; ${ }^{\mathrm{e} D i v i s i o n}$ of Cardiology, Academic Medical Center Amsterdam, Amsterdam, the Netherlands; ${ }^{\mathrm{f}}$ University of Groningen, University Medical Center Groningen, Department of Cardiology, Groningen, the Netherlands; ${ }^{\mathrm{g}} \mathrm{Durrer}$ Center for Cardiovascular Research, ICIN-Netherlands Heart Institute, Utrecht, the Netherlands; and the ${ }^{\mathrm{h}}$ Institute of Cardiovascular Science, Faculty of Population Health Sciences, University College London, London, United Kingdom. *Dr. Mast and Mr. Taha contributed equally to this work and are joint first authors. This work was supported by the Netherlands Cardiovascular Research Initiative, an initiative with support from the Dutch Heart Foundation (CVON2015-12 eDETECT).
} 
A rrhythmogenic right ventricular cardiomyopathy (ARVC) is an inherited cardiomyopathy clinically characterized by ventricular arrhythmias and predominantly right ventricular (RV) dysfunction (1). Typical genetic features of ARVC are reduced penetrance and variable disease expressivity, which complicates family screening (2-4). Comprehensive cardiac screening of family members of patients with ARVC is routinely performed by electrocardiographic (ECG) assessment, Holter monitoring, and cardiac imaging and aims to detect typical ARVC-related abnormalities $(3,5,6)$. However, early ARVC is characterized by a lack of overt structural abnormalities detected by conventional imaging approaches $(3,7)$. Novel imaging techniques could be of incremental value in optimizing ARVC family screening protocols (8).

\section{SEE PAGE 456}

Echocardiographic deformation imaging is a technique that enables the quantification of regional ventricular deformation and provides insight into mechanical synchrony and regional contractility $(9,10)$. Previous reports suggest that this technique is capable of detecting subtle functional abnormalities in the absence of structural abnormalities by conventional imaging (11-13). We recently introduced a new approach that combines multiple deformation parameters into 3 distinct deformation patterns. A clear correlation between abnormal deformation patterns and disease severity among ARVC desmosomal mutation carriers was found (14). In addition, we were able to characterize the underlying electromechanical substrate of these patterns by dedicated computer simulation of deformation patterns. Abnormal deformation was typically seen in the basal area of the RV free wall (or subtricuspid region), which is recognized as 1 of the earliest affected areas in ARVC (12-15). Importantly, abnormal deformation in this specific area was seen during the earliest subclinical stage in which established phenotypic disease expression according to the 2010 task force criteria (TFC) was absent (5). Therefore, echocardiographic deformation imaging may potentially play a pivotal role in improving ARVC family screening.

Although all previously published data were obtained in a cross-sectional study design, this longitudinal study was conducted to explore the value of deformation imaging in screening family members of patients with ARVC. Our hypothesis is that distinct RV deformation abnormalities precede the conventional signs of disease during the early stages of ARVC and can therefore help stratify relatives at risk for disease progression.

\section{METHODS}

STUDY POPULATION. During a 10-year observational period (2006 to 2016), we performed echocardiographic examination according to our ARVC protocol in probands (all fulfilling definite diagnosis according to the 2010 TFC) and their relatives during their clinical work-up for ARVC $(9,16)$. The study participants $(\mathrm{n}=$ 194 , age $>18$ years) were derived from the Dutch national ARVC registry, with patients from University Medical Center Utrecht $(n=161)$, Academic Medical Center Amsterdam $(n=18)$, and University Medical Center Groningen $(n=15)$. Altogether, echocardiographic examinations with appropriate RV recordings for RV deformation imaging were available in 66 ARVC probands and 128 first-degree relatives. All participants were genetically tested for known ARVCrelated pathogenic mutations: plakophilin-2, desmoglein-2, desmocollin-2, desmoplakin, and plakoglobin (5). Nondesmosomal analysis included transmembrane protein 43 and phospholamban $(5,17)$.

The following participants were eligible for this study: 1) first-degree relatives carrying the identical pathogenic ARVC mutation as identified in the probands; and 2) first-degree relatives of mutationnegative probands. These relatives $(n=128)$ were classified according to the presence of subsets of the 2010 TFC during clinical work-up at baseline $(5,14): 1)$ structural stage: relatives fulfilling the 2010 TFC for structural abnormalities detected by echocardiography or cardiac magnetic resonance (CMR); 2) electrical stage: relatives without structural abnormalities fulfilling the 2010 TFC but with ECG abnormalities (repolarization and/or depolarization) and/or history of ventricular arrhythmias as defined by the 2010 TFC; and 3) subclinical stage: relatives without any electrical or structural TFC.

To investigate the value of echocardiographic deformation imaging during the early clinical ARVC

Dr. Asselbergs is supported by a Dekker scholarship (Junior Staff Member 2014To01, Dutch Heart Foundation) and UCL Hospitals NIHR Biomedical Research Centre. Dr. Lumens has received a grant within the framework of the Dr. E. Dekker program of the Dutch Heart Foundation (NHS-2015T082). 
FIGURE 1 Study Design

ARVC imaging registry

with available RV deformation imaging

66 Probands

128 First-degree Relatives

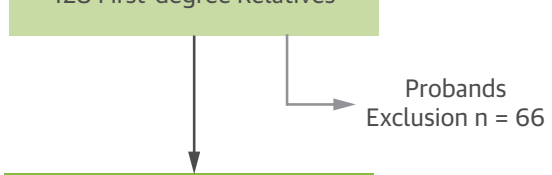

Cardiac imaging

Presence of structural TFC

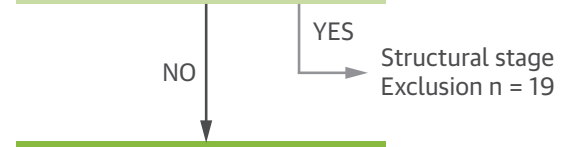

ECG, Holter, History of arrhythmias

Presence of electrical TFC

NO
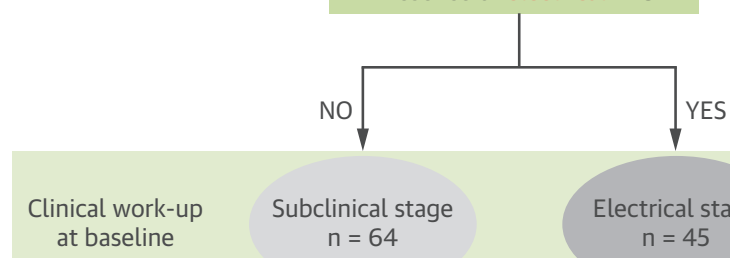

Subclinical stage $n=64$

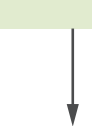

Second

evaluation
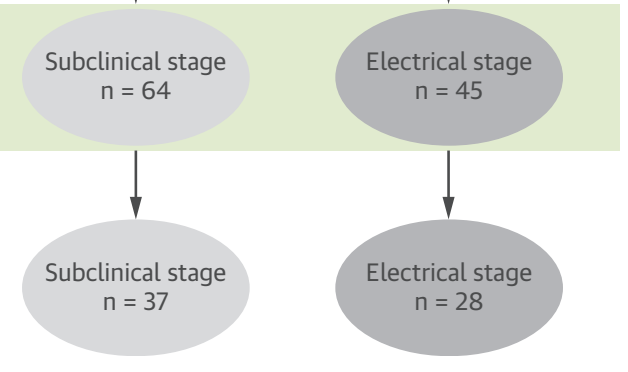

Relatives with available right ventricular (RV) deformation imaging were eligible for this study. The presence of structural abnormalities (as defined by 2010 task force criteria $[T F C]$ ) resulted in exclusion from the study. Only relatives in the early clinical stages (electrical stage and subclinical stage, $n=109$ ) were included. Sixty-five relatives underwent a complete second cardiac evaluation. ARVC = arrhythmogenic right ventricular cardiomyopathy; ECG = electrocardiography.

stages (i.e., subclinical and electrical stages), relatives fulfilling TFC for structural abnormalities (i.e., structural stage) were excluded from further analysis $(n=19)$. Of the remaining 109 early-stage first-degree relatives, a subset of 65 first-degree relatives (60\%) who underwent a second complete cardiac evaluation during follow-up were included in the final study population (Figure 1). The other 44 subjects (40\%) did not undergo a second complete evaluation with all diagnostic modalities during follow-up. Supplemental Table 1 provides a baseline comparison between subjects with follow-up and without complete follow-up. The local medical ethical committees of each participating center approved this study.
CARDIAC EVALUATION. A comprehensive description of the cardiac evaluation is found in the Supplemental Appendix. In brief, all subjects underwent standard 12-lead electrocardiography, which was scored for the presence of repolarization and depolarization abnormalities as defined by the 2010 TFC (5). Holter recordings for $24 \mathrm{~h}$ were analyzed for the presence of ventricular tachycardia and premature ventricular complexes (5). Structural abnormalities as defined by the 2010 TFC were primarily assessed by echocardiography according to standard ARVC protocols $(5,9,16)$. Additionally, left ventricular (LV) involvement was assessed by visual wall motion analysis and measurement of LV ejection fraction using the Simpson biplane method. Additional CMR was performed at the discretion of the treating physician (typically in cases in which echocardiography was of insufficient quality or to verify new abnormalities seen by echocardiography). CMR studies were analyzed for fulfillment of TFC, and LV systolic function was assessed by measurement of $\mathrm{LV}$ ejection fraction $(5,18)$. Contrast-enhanced images after administration of gadolinium were acquired to identify myocardial fibrosis in both the right and left ventricles. Definite diagnosis of ARVC was based on the presence of subsets of the 2010 TFC, which require 2 major criteria, 1 major and 2 minor criteria, or 4 minor criteria (5).

\section{ECHOCARDIOGRAPHIC RV DEFORMATION} IMAGING. All subjects underwent RV echocardiographic deformation imaging using a GE Vivid 7 or a GE Vivid E9 (GE Medical Systems, Milwaukee, Wisconsin) (9). Details on image acquisition and postprocessing are extensively described elsewhere $(9,16)$. In brief, a focused modified narrow-angle 2-dimensional image in the apical 4-chamber view was recorded to assess the RV. Frame rates between 55 and 110 frames/s were accepted for RV deformation imaging. GE EchoPac version 10.2 for PC (GE Healthcare, Little Chalfont, United Kingdom) was used to perform 2-dimensional speckle tracking. After manual tracing, the RV lateral free wall was divided automatically into the basal, mid, and apical segments. Pulmonary valve timing was assessed by Doppler traces in the RV outflow tract obtained in the parasternal short-axis view. The following deformation parameters were measured in the basal area: time to onset of shortening (13), systolic peak strain value $(10,19)$, and post-systolic index (12) (for definitions, see Supplemental Figure 1). These deformation parameters can be combined into 3 distinct deformation patterns, as previously published (Figure 2) (14): type I, defined as normal deformation characterized by onset shortening $\leq 90 \mathrm{~ms}$, systolic peak 
FIGURE 2 Right Ventricular Deformation Patterns



Three distinct deformation patterns are observed in arrhythmogenic right ventricular cardiomyopathy. In a previous report by our group, we used a computer model to simulate type II (middle) pattern by the induction of a mechanical substrate (hypocontractility and increased passive wall stiffness) in the subtricuspid region (14). Type III (right) was simulated by aggravating this substrate. No local pathological electromechanical substrate was present in type I (normal deformation) (left). PVC = pulmonary valve closure; PVO = pulmonary valve opening; RV = right ventricle.

strain $\geq|-20 \%|$, and $\leq 10 \%$ post-systolic shortening; type II, characterized by delayed onset of shortening ( $>90 \mathrm{~ms})$, reduced systolic peak strain $(<|-20 \%|$ and $>|-10 \%|)$, and minor post-systolic shortening ( $>10 \%)$; and type III, characterized by predominantly systolic stretching (systolic peak strain $<|-10 \%|$ ) and major post-systolic shortening.

ARVC DISEASE PROGRESSION. In the final study population of 65 first-degree relatives who underwent 2 separate complete cardiac assessments, disease progression was defined as the presence of a new major or minor task force criterion (structural, depolarization, repolarization, or arrhythmic) that was absent at baseline. RV deformation patterns in the basal area at baseline were evaluated for the predictive value for disease progression.
STATISTICAL ANALYSIS. Data are expressed as mean \pm SD or as median (interquartile range) as appropriate. Normal distribution was tested using the Shapiro-Wilk test. Mean group values were compared using independent Student's $t$-tests or Mann-Whitney $U$ tests as appropriate. Distributions of proportions were performed using Fisher exact tests. Predictive values were expressed as positive predictive value (PPV) and negative predictive value (NPV) with the 95\% confidence interval (CI) calculated using the ClopperPearson method. For interobserver analysis, a second operator performed RV deformation analysis in 20 random subjects. For determination of intraobserver agreement, this sample was reanalyzed by the first observer 6 weeks after the first analysis. Interobserver and intraobserver agreement were determined using linear weighted kappa statistics. Values of $\mathrm{p}<0.05$ 


\begin{tabular}{|c|c|c|c|}
\hline & $\begin{array}{l}\text { Subclinical Stage } \\
\qquad(n=37)\end{array}$ & $\begin{array}{l}\text { Electrical Stage } \\
\qquad(\mathbf{n}=\mathbf{2 8})\end{array}$ & p Value \\
\hline Age, yrs & $26.4 \pm 13.9$ & $39.0 \pm 17.4$ & 0.003 \\
\hline Male & $13(35)$ & $11(39)$ & 0.798 \\
\hline Pathogenic ARVC mutation & $29(78)$ & $25(89)$ & 0.325 \\
\hline PKP2 & $24(65)$ & $21(75)$ & 0.258 \\
\hline DSG2 & $3(8)$ & $1(4)$ & 0.637 \\
\hline$D S P$ & $0(0)$ & $1(4)$ & 0.413 \\
\hline PLN & $2(5)$ & $2(7)$ & 1.00 \\
\hline Symptomatic & $4(11)$ & $4(14)$ & 0.707 \\
\hline Palpitations & $3(8)$ & $4(14)$ & 0.452 \\
\hline Cardiac syncope & 1 (3) & $0(0)$ & 1.00 \\
\hline ARVC definite diagnosis & $0(0)$ & $11(39)$ & $<0.001$ \\
\hline ARVC borderline diagnosis & $0(0)$ & $17(61)$ & $<0.001$ \\
\hline \multicolumn{4}{|l|}{2010 task force criteria } \\
\hline Structural TFC (major/minor) & $0(0)$ & $0(0)$ & 1.00 \\
\hline Depolarization TFC (major/minor) & $0(0)$ & $20(71)$ & $<0.001$ \\
\hline TAD & $0(0)$ & $20(71)$ & $<0.001$ \\
\hline Epsilon wave & $0(0)$ & $0(0)$ & 1.00 \\
\hline Repolarization TFC (major/minor) & $0(0)$ & $9(32)$ & $<0.001$ \\
\hline$T$-wave inversion, leads $V_{1}-V_{2}$ & $0(0)$ & $3(11)$ & 0.075 \\
\hline$T$-wave inversion, leads $V_{1}-V_{3}$ & $0(0)$ & $4(14)$ & 0.030 \\
\hline$T$-wave inversion, leads $V_{4}-V_{6}$ & $0(0)$ & $1(4)$ & 0.431 \\
\hline$T$-wave inversion, leads $V_{1}-V_{6}$ & $0(0)$ & $1(4)$ & 0.431 \\
\hline$T$-wave inversion, leads $V_{1}-V_{4}$, with RBBB & $0(0)$ & $0(0)$ & 1.00 \\
\hline Arrhythmia TFC (major/minor) & $0(0)$ & $13(46)$ & $<0.001$ \\
\hline (Non)sustained VT with superior axis & $0(0)$ & $0(0)$ & 1.00 \\
\hline $\begin{array}{l}\text { (Non)sustained VT with inferior } \\
\text { or unknown axis }\end{array}$ & $0(0)$ & $2(7)$ & 0.182 \\
\hline PVCs $>500 / 24 h$ & $0(0)$ & $12(43)$ & $<0.001$ \\
\hline Family history of TFC (major) & $37(100)$ & $28(100)$ & 1.00 \\
\hline \multicolumn{4}{|l|}{ Echocardiography } \\
\hline RV WMA & 1 (3) & $0(0)$ & 1.00 \\
\hline PLAX RVOT, mm/m² & $15.2 \pm 2.5$ & $15.3 \pm 2.2$ & 0.870 \\
\hline PSAX RVOT, mm/m² & $16.3 \pm 2.8$ & $15.7 \pm 2.4$ & 0.464 \\
\hline RV FAC, \% & $46.4 \pm 6.2$ & $45.7 \pm 7.1$ & 0.691 \\
\hline LVEF, \% & $58.7 \pm 4.6$ & $59.9 \pm 6.2$ & 0.432 \\
\hline CMR & $(n=23)$ & $(n=19)$ & \\
\hline RV WMA & $2(9)$ & $2(11)$ & 1.00 \\
\hline RVEDV, $\mathrm{ml} / \mathrm{m}^{2}$ & $95.9 \pm 14.9$ & $91.9 \pm 8.1$ & 0.055 \\
\hline RVEF, \% & $53.0 \pm 7.2$ & $53.4 \pm 7.6$ & 0.890 \\
\hline LVEF, \% & $56.9 \pm 5.7$ & $56.2 \pm 8.4$ & 0.749 \\
\hline LGE & $2(9)$ & 1 (5) & 1.00 \\
\hline
\end{tabular}

Values are mean $\pm S D$ or $n(\%)$. Definite ARVC diagnosis is defined as the presence of 2 major, 1 major and 2 minor, or 4 minor TFC. Borderline diagnosis of ARVC is defined as the presence of either 1 major and 1 minor or 3 minor TFC.

ARVC $=$ arrhythmogenic right ventricular cardiomyopathy; CMR $=$ cardiac magnetic resonance; $D S G 2=$ desmoglein-2; DSP = desmoplakin; FAC = fractional area change; $L$ GE = late gadolinium enhancement; LVEF left ventricular ejection fraction; PKP2 = plakophilin-2; PLAX = parasternal long-axis view; PLN = phospholamban; $\mathrm{PSAX}=$ parasternal short-axis view; $\mathrm{PVC}=$ premature ventricular complex; $\mathrm{RBBB}=$ right bundle branch block; RVEDV = right ventricular end-diastolic volume; RVEF = right ventricular ejection fraction; RVOT = right ventricular outflow tract; TAD $=$ terminal activation duration; TFC $=$ task force criteria; VT $=$ ventricular tachycardia; $\mathrm{WMA}=$ wall motion abnormality.

\section{RESULTS}

Clinical eVAluation at Baseline. The final study population comprised 65 subjects with a mean age of $31.8 \pm 16.6$ years, of whom 24 (37\%) were male. The majority carried pathogenic mutations ( $\mathrm{n}=54$ [83\%]), which mostly were considered desmosomal mutations ( $\mathrm{n}=50[77 \%]$ ). On the basis of baseline clinical evaluation, 37 subjects were assigned to the subclinical stage, and 28 subjects had electrical abnormalities according to the 2010 TFC (Figure 1). Subjects who showed electrical abnormalities at baseline were significantly older compared with subjects in the subclinical stage $(26.4 \pm 13.9$ years vs. $39.0 \pm 17.4$ years; $\mathrm{p}<0.001)$. Sex and the presence of a pathogenic mutation were not significantly different between subjects in the subclinical and electrical stages (Table 1). At baseline, CMR was available in 23 subjects (62\%) in the subclinical stage and 19 subjects (68\%) in the electrical stage. All mean values of the structural parameters (by CMR and echocardiography) were comparable between subjects in the electrical and subclinical stages (Table 1). At baseline, none of the subjects had signs of LV involvement by echocardiography or CMR. None of the included subjects had histories of sustained ventricular arrhythmias, and none were on antiarrhythmic medications. Eight subjects (12\%) had implantable cardiac-defibrillators placed at baseline for primary prevention ( 3 in the subclinical stage and 5 in the electrical stage). No baseline differences were seen between subjects who underwent a second evaluation $(n=65)$ and those who did not $(n=44)$ (Supplemental Table 1).

RV DEFORMATION PATTERNS IN RELATIVES AT BASELINE. In the final study population, type I deformation was seen in 28 subjects (43\%), type II deformation was seen in 33 subjects $(51 \%)$, and type III deformation was seen in 4 subjects (6\%). Abnormal deformation (types II and III) was more frequently seen in older subjects $(36.6 \pm 7.2$ years vs. $25.5 \pm 13.7$ years; $\mathrm{p}=0.005)$ and in pathogenic mutation carriers $(92 \%$ vs. $71 \%$; $p=0.045)$. Sex was equally distributed between subjects with normal and abnormal deformation patterns (male $36 \%$ vs. $38 \%$; $\mathrm{p}=1.00$ ).

The 37 subjects in the subclinical stage were mainly characterized by deformation pattern type I ( $\mathrm{n}=23$ [62\%]), whereas deformation pattern type II was seen in the remaining 14 subjects (38\%). The electrical stage was mainly characterized by deformation pattern type II $(n=19$ [68\%]). In the remaining subjects in the electrical stage, type I was seen in were considered to indicate statistical significance. All statistical analyses were performed using commercially available software (SPSS Statistics for Windows version 21.0, IBM, Armonk, New York). 


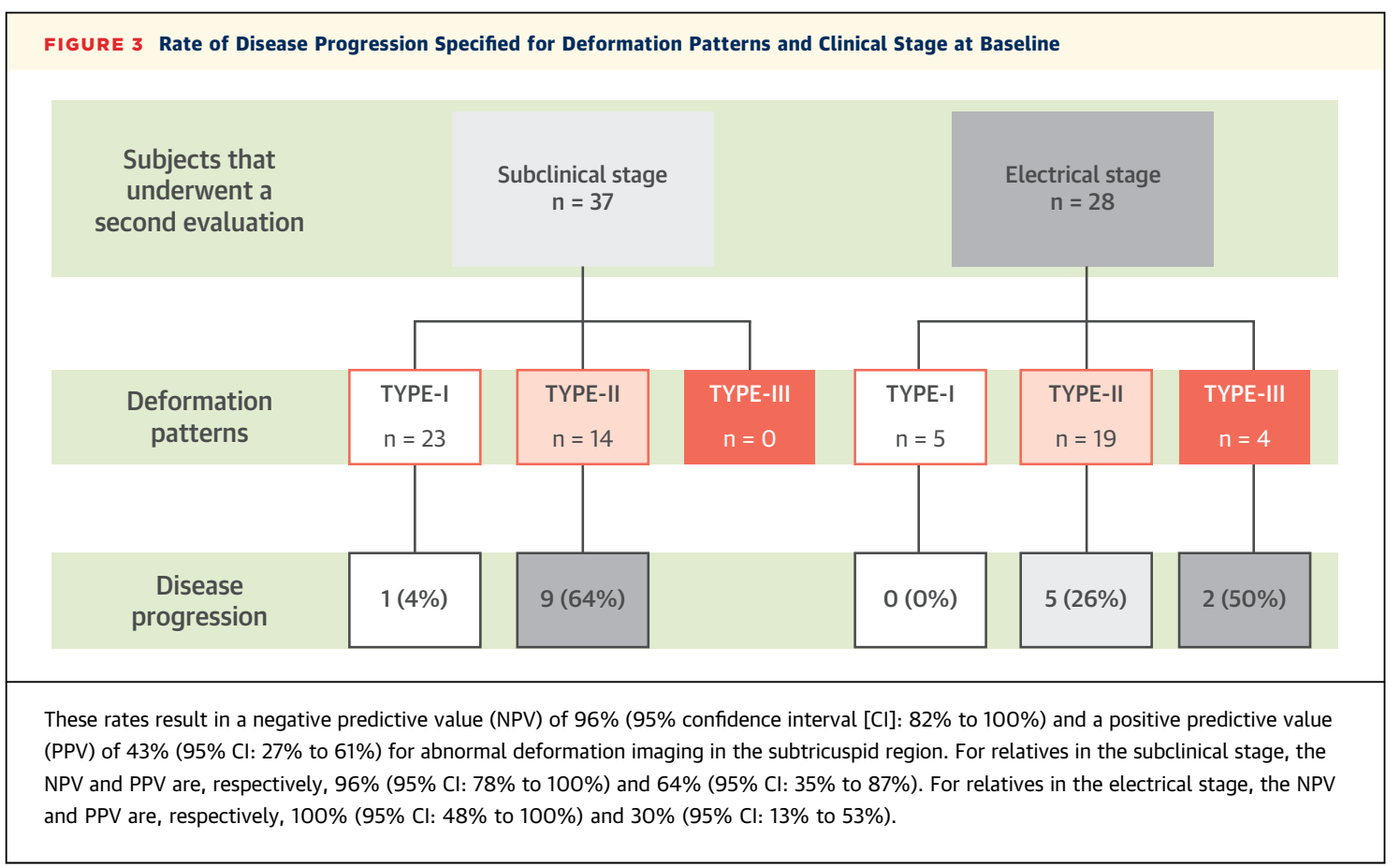

$5(18 \%)$ and type III in $4(14 \%)$. The distribution of baseline deformation patterns specified for the presence of ECG abnormalities as defined by the TFC is shown in Supplemental Table 2.

Inter-rater and intrarater reproducibility for RV deformation pattern classification was high, respectively 0.94 (95\% CI: 0.81 to 1.00 ) and 0.93 (95\% CI: 0.79 to 1.00$)$

DISEASE PROGRESSION. The mean follow-up duration was $3.7 \pm 2.1$ years and was equally distributed between subjects who showed signs of disease progression and those without signs of disease progression (respectively, $4.5 \pm 2.0$ vs. $3.5 \pm 2.1$ years; $\mathrm{p}=0.09)$. Altogether, 17 relatives $(26 \%)$ showed signs of ARVC disease progression.

Electrical progression occurred more frequently compared with structural progression: 11 subjects showed only electrical progression, 4 subjects showed electrical progression along with structural progression, and 2 subjects showed structural progression on top of pre-existing electrical disease at baseline. None of the subjects experienced a sustained arrhythmic event or appropriate implantable cardioverterdefibrillator intervention during follow-up.

The progression rates among the carriers of different mutations are shown in Supplemental Figure 2.

PREDICTIVE VALUE OF ABNORMAL DEFORMATION IN EARLY ARVC. Of the 28 subjects with a normal deformation pattern (type I) at baseline, only 1 subject showed disease progression, expressed as an increased premature ventricular complex count of $>500$ over $24 \mathrm{~h}$ during second evaluation. In the 37 subjects with abnormal deformation patterns (types II and III) at baseline, disease progression was seen in 16 subjects (43\%). The NPV of normal deformation at baseline for disease progression was 96\% (95\% CI: $82 \%$ to $100 \%$ ). The PPV of abnormal deformation at baseline for disease progression was $43 \%$ (95\% CI: $27 \%$ to $61 \%$ ). The predictive values were similar in a subcohort consisting only of mutation-positive relatives ( $n=54)$ : NPV 95\% (95\% CI: $75 \%$ to $100 \%$ ) and PPV $44 \%$ (95\% CI: $27 \%$ to $62 \%$ ).

Figure 3 shows a flowchart of the rate of disease progression specified for both deformation pattern and clinical stage at baseline. Among the 23 subjects in the subclinical stage with normal deformation at baseline, only 1 subject showed disease progression (NPV 96\%; 95\% CI: $78 \%$ to $100 \%$ ). Among the 14 subjects in the subclinical stage with abnormal (type II) deformation, 9 (64\%) showed disease progression (Figure 3). This resulted in a PPV of 64\% (95\% CI: 35\% to $87 \%)$.

Among the 5 electrical-stage subjects with normal deformation at baseline, disease progression occurred in none (NPV 100\%; 95\% CI: 48\% to $100 \%$ ). Of the 19 subjects in the electrical stage with type II pattern at baseline, 5 (26\%) showed signs of disease progression. Two of the 4 subjects $(50 \%)$ in the electrical stage with deformation pattern type III showed 
disease progression. The PPV of abnormal deformation (type II or III pattern) on disease progression in the electrical stage was $30 \%$ (95\% CI: $13 \%$ to $53 \%$ ).

\section{DISCUSSION}

The main findings of our study are that in case of normal findings on conventional echocardiography and CMR: 1) first-degree relatives of patients with ARVC with normal deformation in the RV basal area did not show disease progression during a mean follow-up of nearly 4 years; and 2) the presence of abnormal deformation at baseline was associated with unequivocal signs of disease progression during follow-up in early ARVC. The results of this study might have implications for our follow-up strategy of relatives in clinical practice. Relatives with normal RV deformation on top of normal results during standard cardiac screening seem to have an excellent midterm prognosis, and less frequent cardiac screening might be equally effective.

NORMAL RV DEFORMATION IMAGING IN EARLY ARVC. Our study shows that deformation imaging is able to identify relatives at low risk for disease progression. This holds true particularly for relatives in the earliest stage without any established disease expression as defined by the TFC. Normal deformation in the RV basal area in addition to the absence of abnormalities detected by electrocardiography, Holter monitoring, and conventional cardiac imaging largely excludes disease progression for at least almost 4 years. Previously, we demonstrated with computer modeling that deformation pattern type I represents normal electromechanical properties of the RV myocardium such as seen in healthy subjects (14). We focused on the RV basal area (subtricuspid region) because previous studies have convincingly shown that this area is 1 of the first affected regions in ARVC $(12,13,15)$. Our results suggest that relatives without any disease expression (including normal deformation in the subtricuspid region) are in a clinical stage that precedes the subclinical stage. Traditionally, a clinical stage without any disease expression in ARVC is often considered as the concealed stage (1). Our data show that deformation imaging helps discriminate between relatives who are in a true concealed stage and relatives with subtle local RV mechanical dysfunction not detected by conventional approaches (subclinical stage) (Figure 4). A recent consensus statement by an international task force recommends repeated clinical assessment in all family members of patients with ARVC every 2 to 3 years, even in those without any morphological or functional abnormalities (6). In the present study, we observed low progression rates in relatives of patients with ARVC in the true concealed stage (i.e., relatives with normal deformation in the subtricuspid region in addition to normal findings by conventional techniques). This allows us to speculate that the follow-up interval in this group might be extended beyond the current recommendations (6). However, further studies with longer follow-up and preferentially larger patient numbers, in which disease progression is accurately assessed by CMR, are needed to further substantiate our findings. Moreover, individual factors (e.g., cardiac symptoms and sports activity) should always be considered when determining individual follow-up intervals.

ABNORMAL RV DEFORMATION IMAGING IN EARLY ARVC. By definition, relatives in the subclinical stage lack any established disease expression as defined by the TFC. Interestingly, one-third of the included subclinical staged subjects in this study were identified with an abnormal deformation pattern (type II). In a recent study, we showed that this abnormal deformation pattern was present in almost one-half of the desmosomal mutation carriers in the subclinical stage, and the underlying electromechanical substrate seems to be regional hypocontractility and mildly increased passive wall stiffness (14). This finding was confirmed in the present study, in which abnormal deformation patterns were encountered in subclinical-stage subjects without any established disease expression, including the absence of ECG abnormalities (Supplemental Table 2). One of our main findings was that the presence of abnormal deformation actually precedes ECG abnormalities, as approximately onehalf of the subjects in the subclinical stage with abnormal deformation developed unequivocal signs of disease progression during follow-up, primarily electrical disease progression. The association between the presence of abnormal deformation and the occurrence of established disease expression during follow-up supports our hypothesis that the observed deformation patterns are a functional representation of an underlying pathological electromechanical substrate.

The subjects in our cohort did not experience any life-threatening events such as sudden cardiac death, sustained ventricular arrhythmia, or appropriate implantable cardioverter-defibrillator intervention during follow-up. This could be explained by the fact that all relatives fulfilling structural TFC were excluded from our study, while especially this form of disease expression is seen in all relatives prior to 
FIGURE 4 Suggested Follow-Up Strategy in Relatives Depends on Clinical Stage of Arrhythmogenic Right Ventricular Cardiomyopathy

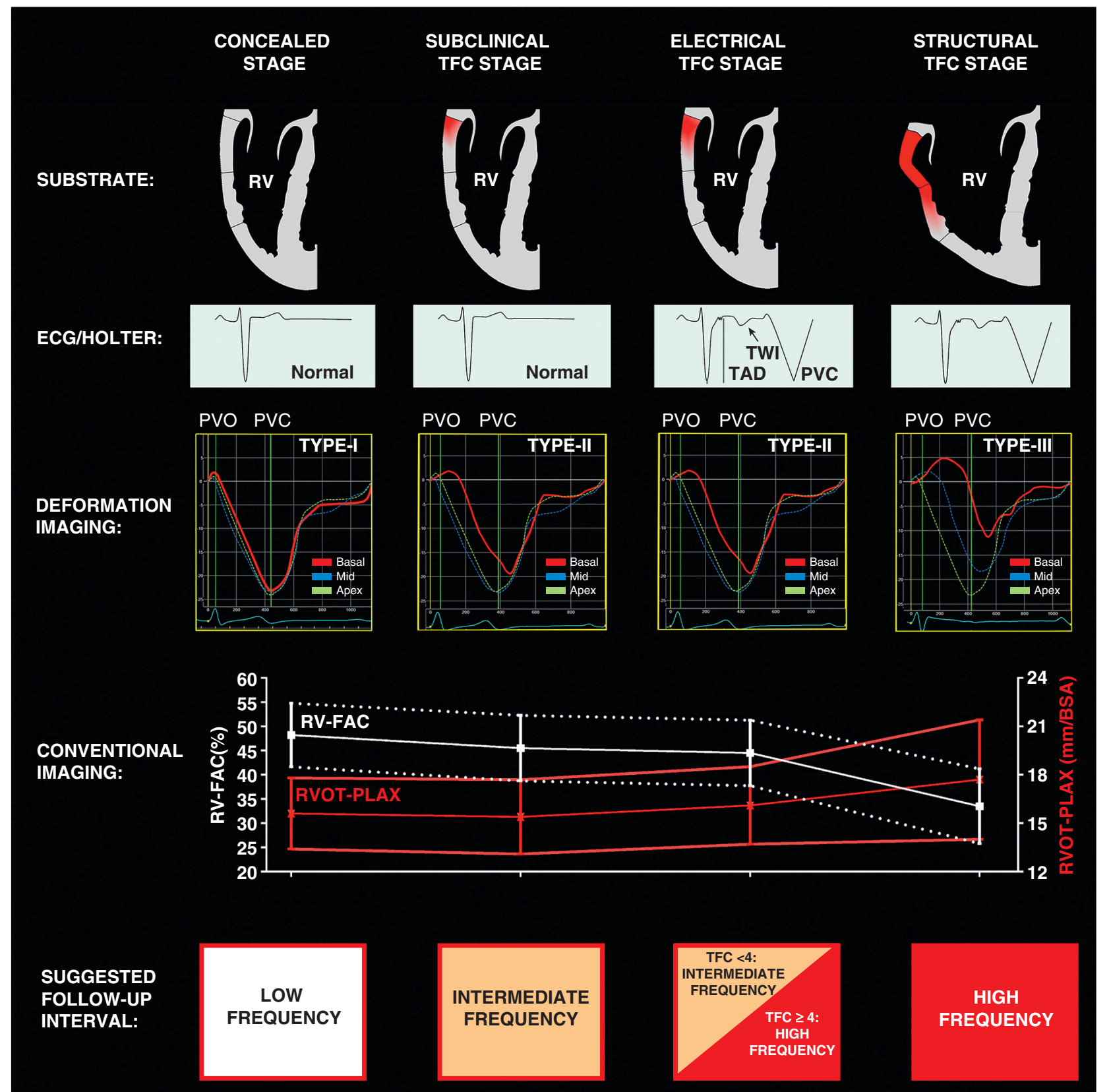

In the concealed stage, right ventricular deformation imaging shows normal deformation (type I), suggesting the absence of electromechanical substrate. In the subclinical stage, right ventricular deformation imaging shows an abnormal pattern, but electrical and structural abnormalities (as defined by the task force criteria [TFC]) are not detectable. The electrical stage is characterized by electrical abnormalities, and deformation imaging in this stage shows a transition between type II and type III deformation patterns. In the structural stage, there are both electrical and structural abnormalities as defined by the TFC, and deformation imaging in this stage shows type III deformation, which is associated with a large right ventricular electromechanical substrate. Normal deformation (type I) without any other detected abnormalities excludes the presence of an electromechanical substrate, and follow-up intervals in this stage might be less frequent compared with the follow-up strategies recommended by current guidelines (6). BSA = body surface area; ECG = electrocardiography; FAC = fractional area change; PLAX = parasternal long-axis view; PVC = premature ventricular complexes; RV = right ventricle; RVOT = right ventricular outflow tract; TAD $=$ terminal activation duration; TWI $=$ T-wave inversion. 
sustained arrhythmic events $(3,4,7)$. Another explanation could be that this cohort was too small, and the lack of events was possibly a matter of chance. Although we were not able to prove any association between abnormal deformation and sustained arrhythmias, we do speculate that abnormal deformation is an early sign of structural changes. Considering the apparent low arrhythmic risk in patients with no structural expression, cardiac screening every 2 years in accordance with the current task force consensus statement seems to be sufficient and safe (6) (Figure 4).

TOWARD OPTIMIZATION OF FAMILY SCREENING PROTOCOLS. To our best knowledge, the present study is the first to prospectively investigate the prognostic value of RV deformation imaging in early ARVC. A recent retrospective study by Leren et al. (20) reported a multimodality approach in identifying subjects at risk for ventricular arrhythmias during early ARVC and thereby aiming at the use of deformation imaging in addition to conventional techniques. Our study is in line with their multimodality design during family screening and further highlights the additional value of $\mathrm{RV}$ deformation imaging in ARVC.

A recent expert consensus document of the European Heart Association supports the additional use of strain echocardiography in the echocardiographic assessment of ARVC, particularly in early ARVC, when the diagnosis is challenging (8). We may be entering a new era in which echocardiographic deformation imaging will participate in the field of clinical decision making in ARVC (21).

STUDY LIMITATIONS. On the basis of the rates of disease progression that were observed in our cohort after almost 4 years, we made suggestions for followup intervals for family members of patients with ARVC. However, these intervals may not be suitable for all family members. First, it is known that the disease behaves differently among the carriers of different mutations, while our cohort represented mainly plakophilin-2 and phospholamban mutation carriers (17). Additionally, in our proposed follow-up intervals, we did not take into consideration factors such as age, sex, presence of cardiac symptoms, and sports activity $(4,22)$. These factors may have a significant influence on disease progression and thus should be taken into consideration in studies aiming to make recommendations for follow-up intervals. Even though the present study included a relatively large cohort of patients with this relatively rare disease, our study population was too small to correct for genetic profile and additional clinical factors in a multivariate analysis.

Forty percent of the baseline cohort could not be included in the study, because their second evaluation did not take place during our study period or because the second evaluation did not include all diagnostic modalities that are needed to adequately assess disease progression. On the basis of the baseline comparison between subjects with follow-up and without available follow-up (Supplemental Table 1), relevant selection bias seems unlikely.

Structural disease progression was primarily assessed using conventional echocardiography, and 25 subjects (38\%) underwent additional CMR. However, the sensitivity of conventional echocardiography is known to be inferior to that of CMR, which could potentially lead to lower detection of structural abnormalities in subjects who did not undergo CMR (23). In future studies, disease progression should be accurately assessed using both CMR and echocardiography.

\section{CONCLUSIONS}

Echocardiographic deformation imaging is capable to identify relatives who are at low risk for disease progression during the early stages of ARVC. A normal RV deformation pattern at baseline is associated with an absence of disease progression during midterm follow-up in relatives of patients with ARVC, suggesting that a low-frequency follow-up strategy would suffice. Moreover, the presence of abnormal $\mathrm{RV}$ deformation in early ARVC is associated with unequivocal signs of disease progression. Therefore, our data suggest that echocardiographic deformation imaging may potentially be implemented in ARVC family screening protocols. Future studies including a larger study population are required to validate our data.

ADDRESS FOR CORRESPONDENCE: Dr. ArCO J. Teske, Department of Cardiology, Division Heart and Lungs, University Medical Center Utrecht, Heidelberglaan 100, 3584CX Utrecht, the Netherlands. E-mail: a.j.teske-2@umcutrecht.nl. 


\section{PERSPECTIVES}

COMPETENCY IN MEDICAL KNOWLEDGE: The present study demonstrates that in the absence of structural TFC, normal echocardiographic deformation in the subtricuspid region identifies family members of patients with ARVC who are at low risk for disease progression. Abnormal echocardiographic deformation in this region is associated with unequivocal signs of disease progression.

TRANSLATIONAL OUTLOOK: Future studies including a larger number of family members of patients with ARVC and with longer follow-up are required to validate the predictive value of echocardiographic deformation imaging in risk stratification in early ARVC. Echocardiographic deformation imaging may become an important part of family screening protocols in ARVC. We should be heading in the direction of a predictive model in which a variety of clinical parameters are implemented, to create individual, tailor-made follow-up strategies for family members of patients with ARVC.

\section{REFERENCES}

1. Corrado D, Link MS, Calkins $H$, et al. Arrhythmogenic right ventricular cardiomyopathy. N Engl J Med 2017;376:61-72.

2. Nava $A$, Bauce $B$, Basso C, et al. Clinical profile and long-term follow-up of 37 families with arrhythmogenic right ventricular cardiomyopathy. J Am Coll Cardiol 2000;36:2226-33.

3. Te Riele AS, James CA, Rastegar $N$, et al. Yield of serial evaluation in at-risk family members of patients with ARVD/C. J Am Coll Cardiol 2014;64: 293-301.

4. Te Riele AS, James CA, Groeneweg JA, et al, Approach to family screening in arrhythmogenic right ventricular dysplasia/cardiomyopathy. Eur Heart J 2016;37:755-63.

5. Marcus FI, McKenna WJ, Sherrill D, et al. Diagnosis of arrhythmogenic right ventricular cardiomyopathy/dysplasia: proposed modification of the task force criteria. Circulation 2010;121:1533-41.

6. Corrado $\mathrm{D}$, Wichter $\mathrm{T}$, Link MS, et al. Treatment of arrhythmogenic right ventricular cardiomyopathy/dysplasia: an international task force consensus statement. Eur Heart J 2015;36:3227-37.

7. Te Riele AS, Bhonsale $A$, James CA, et al. Incremental value of cardiac magnetic resonance imaging in arrhythmic risk stratification of arrhythmogenic right ventricular dysplasia/ cardiomyopathy-associated desmosomal mutation carriers. J Am Coll Cardiol 2013;62:1761-9.

8. Haugaa $\mathrm{KH}$, Basso $\mathrm{C}$, Badano $L P$, et al, Comprehensive multi-modality imaging approach in arrhythmogenic cardiomyopathy-an expert consensus document of the European Association of Cardiovascular Imaging. Eur Heart J Cardiovasc Imaging 2017;18:237-53.

9. Teske AJ, De Boeck BW, Melman PG, Sieswerda GT, Doevendans PA, Cramer MJ. Echocardiographic quantification of myocardial function using tissue deformation imaging, a guide to image acquisition and analysis using tissue
Doppler and speckle tracking. Cardiovasc Ultrasound 2007;5:27.

10. Teske AJ, Cox MG, De Boeck BW, Doevendans PA, Hauer RN, Cramer MJ. Echocardiographic tissue deformation imaging quantifies abnormal regional right ventricular function in arrhythmogenic right ventricular dysplasia/cardiomyopathy. J Am Soc Echocardiogr 2009;22: 920-7.

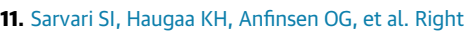
ventricular mechanical dispersion is related to malignant arrhythmias: a study of patients with arrhythmogenic right ventricular cardiomyopathy and subclinical right ventricular dysfunction. Eur Heart J 2011;32:1089-96.

12. Teske AJ, Cox MG, Te Riele AS, et al. Early detection of regional functional abnormalities in asymptomatic ARVD/C gene carriers. J Am Soc Echocardiogr 2012;25:997-1006.

13. Mast TP, Teske AJ, Te Riele AS, et al. Prolonged electromechanical interval unmasks arrhythmogenic right ventricular dysplasia/cardiomyopathy in the subclinical stage. J Cardiovasc Electrophysiol 2016;27(3):303-14.

14. Mast TP, Teske AJ, Walmsley J, et al. Right ventricular deformation imaging and computer simulation for electromechanical substrate characterization in arrhythmogenic right ventricular cardiomyopathy (ARVC). J Am Coll Cardiol 2016; 68:2185-97.

15. Te Riele AS, James CA, Philips $B$, et al. Mutation-positive arrhythmogenic right ventricular dysplasia/cardiomyopathy: the triangle of dysplasia displaced. J Cardiovasc Electrophysiol 2013:24:1311-20.

16. Mast TP, Teske AJ, Doevendans PA, Cramer MJ. Current and future role of echocardiography in arrhythmogenic right ventricular dysplasia/cardiomyopathy. Cardiol J 2015:22: 362-74.
17. Groeneweg JA, van der Zwaag PA, Olde Nordkamp LR, et al. Arrhythmogenic right ventricular dysplasia/cardiomyopathy according to revised 2010 task force criteria with inclusion of non-desmosomal phospholamban mutation carriers. Am J Cardiol 2013;112:1197-206.

18. Tandri $H$, Calkins $H$, Nasir $K$, et al. Magnetic resonance imaging findings in patients meeting task force criteria for arrhythmogenic right ventricular dysplasia. J Cardiovasc Electrophysiol 2003; 14:476-82.

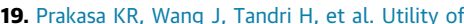
tissue Doppler and strain echocardiography in arrhythmogenic right ventricular dysplasia/cardiomyopathy. Am J Cardiol 2007;100:507-12.

20. Leren IS, Saberniak J, Haland TF, Edvardsen T, Haugaa KH. Combination of ECG and echocardiography for identification of arrhythmic events in early ARVC. J Am Coll Cardiol Img 2017;10:503-13.

21. Teske AJ, Mast TP. Moving from multimodality diagnostic tests toward multimodality risk stratification in ARVC. J Am Coll Cardiol Img 2017;10: 514-7.

22. Sawant $A C$, Calkins H. Relationship between arrhythmogenic right ventricular dysplasia and exercise. Card Electrophysiol Clin 2015;7:195-206.

23. Borgquist R, Haugaa $K H$, Giljam $T$, et al. The diagnostic performance of imaging methods in ARVC using the 2010 task force criteria. Eur Heart J Cardiovasc Imaging 2014;15:1219-25.

KEY WORDS arrhythmogenic right ventricular cardiomyopathy, ARVD/C deformation imaging, disease progression, family screening, strain imaging

APPENDIX For supplemental methods, tables, and figures, please see the online version of this paper. 